LOUIS BOUSQUET

ORCID: 0000-0003-1069-999

University of Hawai'i at Mānoa

lb2010@hawaii.edu

\title{
L'ISLAM DANS LES ROMANS DE MICHEL HOUELLEBECQ
}

Le regard de Michel Houellebecq envers la religion musulmane a beaucoup évolué dans son œuvre romanesque. Traité avec mépris et condescendance dans ses premiers textes, l'islam se transforme en une alternative possible à la névrose des sociétés modernes dans son roman Soumission. En suivant chronologiquement ces changements de perspective, j'essaierai d'illustrer l'opportunisme lucide du romancier qui poursuit avec insistance une tâche difficile, trouver une alternative satisfaisante à l'existence douloureuse de ses héros. Ces derniers sont les représentants d'une catégorie sociologique multiple qui se décline tout au long des romans ; nous les nommerons tour à tour, mâles dominés, hommes bêtas ${ }^{1}$, homoncules $^{2}$ ou encore incels ${ }^{3}$ selon leur incarnation respective. Ils seront finalement transformés, comme par magie et avec un certain sens de la dérision, grâce aux " mécanismes de la servitude volontaire ${ }^{4}$, en mâles dominants. Je soulignerai

${ }^{1}$ En opposition à « l'animal oméga », titre d'un chapitre des Particules Élémentaires.

2 «Homonculus touristicus est un individu en fuite, il s'ennuie, il s'évade, il s'échappe. Rien ne peut le faire dévier de son besoin de foutre le camp » (O. Bardolle, De la prolifération des homoncules, l'Esprit des Péninsules, Dijon-Quetigny 2008, p. 96).

${ }^{3}$ Ce qualificatif est discutable, dans un article du New York Times du 12 juillet 2018, Adam Hirsch qualifie Raphaël Tisserand, personnage de L'Extension du Domaine de la Lutte, de «proto-incel », et fait de Houellebecq un visionnaire quant à ce phénomène des « involuntary celibate » (A. Kirsch, «A French Novelist Imagined Sexual Dystopia. Now It's Arrived », <https://www.nytimes. com/2018/07/12/books/review/michael-houellebecqs-sexual-distopia.html?smprod=nytcore-ipad\&smid=nytcore-ipad-share $>$ [consulté le 20.07.2018]).

${ }^{4}$ M. Onfray, Miroir du Nihilisme, Galilée, Paris 2017, p. 48. 
l'art subtil de la récupération de Michel Houellebecq qui utilise l'islam politique à son profit, comme une ultime stratégie de consommation, et en conclusion, comme faire-valoir artistique. Notre postulat découvre la manipulation idéologique de l'écrivain qui transforme une angoisse sous-jacente, présente dans une partie de la société française, en opportunité paradoxale et exceptionnelle, car inscrite dans une logique propre au roman ${ }^{5}$. Ce travail sera basé sur la lecture exclusive de quatre romans ${ }^{6}$ de l'écrivain qui traitent de la religion musulmane : Les Particules Élémentaires ${ }^{7}$, La Possibilité d'une île ${ }^{8}$, Plateforme ${ }^{9}$ et Soumission ${ }^{10}$.

\section{L'ISLAM, REBUT DES RELIGIONS}

La première véritable mise en cause de l'islam dans l'œuvre romanesque de Michel Houellebecq, sera trouvée dans Les Particules Élémentaires. Si l'islam est une question périphérique à la problématique de l'ouvrage en général, il est cependant associé à deux problèmes majeurs que l'auteur va tenter de résoudre : le devenir des sociétés contemporaines occidentales qui selon le narrateur sont désormais dépourvues de religion, et plus précisément la solitude affective et physiologique qui s'abat cruellement sur les héros du roman. La religion musulmane est violemment attaquée lors d'une discussion qui met en scène deux personnages, en l'occurrence deux homoncules, c'est-à-dire deux hommes esseulés qui souffrent avec plus ou moins d'acuité de cet isolement social involontaire :

${ }^{5}$ C'est ainsi que contrairement à de nombreux critiques de l'œuvre de Michel Houellebecq, nous considérons les épithètes d'islamophobe voire d'islamophile comme hors sujet; l'auteur selon notre lecture est plutôt « islamo-opportuniste ». Shlomo Sand, par exemple, caractérise Soumission comme " [1'] un des ouvrages les plus manifestement islamophobes, édités en France au début du $\mathrm{XXI}^{\mathrm{e}}$ siècle : par sa dimension d'épouvante » (S. Sand, La fin de l'intellectuel français ?, Cahiers Libres, Éditions La Découverte, Paris 2016 [Kindle Edition], p. 4773). Nous refusant à toute lecture psychologisante de l'œuvre de Michel Houellebecq, nous soulignons dans ce travail que « l'épouvante » dans le texte est principalement celle de l'homoncule déprimé qui voit se profiler une vie de solitude douloureuse. Cette « épouvante » n'est jamais causée par une religion qui sied parfaitement aux intérêts de François et génère, de façon plus large, l'adhésion, sinon la soumission générale des clercs, les universitaires et les politiques du roman.

${ }^{6}$ Ces textes se distinguent du reste de son œuvre par l'intérêt qu'ils portent à la religion en général et à l'islam en particulier.

${ }^{7}$ M. Houellebecq, Les Particules Élémentaires, J'ai Lu, Paris 2001. Le terme « islam » est mentionné à quatre reprises dans ce roman.

${ }^{8}$ M. Houellebecq, La Possibilité d'une île, Fayard, Paris 2005. Il existe onze mentions du terme dans cet ouvrage avec des variantes notables telles « islamistes », « islamophobe » ou encore « islamique ».

${ }^{9}$ M. Houellebecq, Plateforme, Flammarion, Mesnil-sur-L'Estrée 2001. L'auteur utilise dans Plateforme le terme et ses déclinaisons à quinze reprises.

${ }^{10}$ M. Houellebecq, Soumission, Flammarion, Mesnil-sur-L'Estrée 2015. Soumission est centré autour de la question de l'islam politique, le livre comporte soixante-sept mentions de cette famille de mots. 
il-y-a Desplechin ${ }^{11}$, le directeur du département biologie du CNRS et Michel Djerzinsky, scientifique lui aussi et l'un des principaux protagonistes de l'histoire. Desplechin prophétise la mort de toutes les religions au nom d'une prémisse scientifique incontournable : « le besoin de certitude rationnelle ${ }^{12}$. Cette nécessité résume selon lui tout le mouvement intellectuel de la civilisation occidentale et mène aujourd'hui à son déclin. À l'aune de ce critère scientifique absolu, toutes les religions traditionnelles sont vouées à un effacement inexorable :

je sais bien que l'islam - de loin la plus bête, la plus fausse et la plus obscurantiste de toutes les religions - semble actuellement gagner du terrain ; mais ce n'est qu'un phénomène superficiel et transitoire : à long terme l'islam est condamné, encore plus sûrement que le christianisme ${ }^{13}$.

Nous trouvons en filigrane dans cette condamnation récurrente, l'idéologie progressiste et scientiste héritée du XVIII ${ }^{\mathrm{e}}$ et $\mathrm{XIX}^{\mathrm{e}}$ siècle. Ce réquisitoire brutal laisse deviner une angoisse sourde et lancinante qui trouve son origine dans l'aspect le plus sociohistorique de la réflexion houellebecquienne. Car la perte de la religion n'est pas simplement ici celle d'une métaphysique par ailleurs largement étrangère aux personnages de Michel Houellebecq ; c'est principalement la disparition d'un système, vecteur de cohésion et de protection, maintenant sacrifié ${ }^{14}$ à l'autel de la poursuite scientifique. Desplechin prophétise ainsi dans sa diatribe contre l'islam, non seulement la disparition des religions, mais aussi celle des sociétés modernes. Le raisonnement philosophique et sociologique de Michel Houellebecq trouve son inspiration chez les penseurs socialistes ${ }^{15} \mathrm{du} \mathrm{XIX}{ }^{\mathrm{e}}$ siècle et plus précisément à travers la réflexion d'Auguste Comte. Comte mieux que quiconque, a pressenti l'importance de la religion ${ }^{16}$ dans le devenir des peuples occidentaux : elle est pour le sociologue à l'essence des sociétés, puisqu'elle forme leur infrastructure. C'est ainsi que nous pouvons comprendre la gravité de cette question lancinante posée par le romancier en forme de leitmotiv : « mais combien de temps la société occidentale pourrait-elle subsister sans une religion ? ${ }^{17}$. Pour survivre, Houellebecq à travers ses personnages nous répète que toute société doit reposer sur un système religieux ${ }^{18}$

${ }^{11}$ Personnage dont nous ne connaîtrons jamais le prénom.

12 M. Houellebecq, Les Particules..., p. 270.

13 Ibidem, p. 271.

14 « À ce besoin de certitude rationnelle, l'Occident aura finalement tout sacrifié : sa religion, son bonheur, ses espoirs, et en définitive sa vie » (ibidem, p. 270).

15 «C'est à l'automne 1830, en réaction contre l'ultralibéralisme [...] qu'apparut pour la première fois la nébuleuse d'idées que Pierre Leroux nommera en 1834 socialisme » (B. Viard, Les Tiroirs de Michel Houellebecq, Puf, Mayenne, 2013, p. 55).

16 « Aussi paradoxal que cela puisse paraître, c'est bien une religion qui couronne le système positiviste. En effet, seule une religion possède assez de force unificatrice pour rassembler les hommes autour d'une visée commune universelle et pour harmoniser en chacun le cœur et la raison » (B. Jolibert, Science et Religion chez Auguste Comte, <https://espe.univreunion.fr/fileadmin/ Fichiers/ESPE/bibliotheque/expression/23/Comte.pdf $>$ ).

${ }_{17}$ M. Houellebecq, Les Particules..., p. 162.

${ }^{18}$ Qui en essence relie les hommes entre eux, selon Auguste Comte. 
qui génère une organisation morale et organique entre ceux qui la composent. Les homoncules, Michel son frère Bruno et accessoirement ici l'homosexuel solitaire Desplechin, apparaissent comme les symptômes souffrants d'une société qui ne fonctionne plus. Michel Djerzinski tente, suivant les préceptes d'Auguste Comte, d'utiliser la science comme un outil transcendant, associée ici à une nouvelle religion improbable, elle pourrait permettre de fédérer sous une bannière protectrice une population aliénée : «Si la science fut, au moyen âge, essentiellement soumise à la religion de dieu, elle doit désormais, au nom de la raison et de la morale, servir, beaucoup plus complètement, la religion de l'humanité ${ }^{19}$. Mais au lieu d'inventer un nouveau culte comme son prédécesseur, Houellebecq par le biais de Djerzinski crée de nouveaux disciples ${ }^{20}$. Une post-humanité de clones jouisseurs qui se ressemble telle une secte de mêmes ${ }^{21}$. L'islam dans ce contexte est présenté comme un épouvantail symbolique, la version la plus primitive et la plus obscurantiste des trois grandes religions monothéistes à l'ère matérialiste. Cependant, l'opposition des religions au progrès scientifique est désormais dérisoire et dépassée, car l'homme moderne chez Houellebecq est devenu son propre démiurge et il n'a que faire de tels systèmes perçus comme obsolètes et dangereux. Dans le projet du clonage aux contours volontairement flous, c'est la tentation hédoniste ${ }^{22}$, précisément le fruit d'un individualisme forcené, qui va à l'encontre du message moral et social des religions. Le caractère méprisant et parfois provocant des déclarations des personnages envers l'islam s'explique dans cette quête viscéralement égoïste et désespérée qui mène au plaisir individuel. Car l'hédonisme, chez Michel Houellebecq, se présente comme la dernière alternative possible qui reste à l'homoncule pour essayer de survivre à l'atomisation ${ }^{23}$ traumatique des sociétés occidentales. La réponse de Desplechin à la question de Djerzinski est à ce sujet parfaitement symptomatique : «Qu'est-ce que vous allez faire, maintenant ? [...] Voyager... Un peu de tourisme sexuel, peut-être $»^{24}$. Cette réplique nonchalante et cynique en forme de programme existentiel se heurtera au terrorisme islamiste dans Plateforme.

${ }^{19}$ A. Comte, Système de politique positive, ou Traité de sociologie instituant la Religion de l'Humanité, Larousse, Paris 1890, p. 746.

20 «[...] l'humanité devait disparaître, l'humanité devait donner naissance à une nouvelle espèce, asexuée et immortelle, ayant dépassé l'individualité, la séparation et le devenir » (M. Houellebecq, Les Particules..., p. 308).

21 «[...] tous les individus seraient porteurs du même code génétique » (ibidem, p. 312).

22 Voir l'article de Bruno sur le projet social utopique Marseillan Plage et les « éléments minoritaires » et autres « délinquants arabes » (ibidem, p. 223). Par ailleurs, en conclusion du roman, le narrateur suggère de cloner sur la peau de la nouvelle humanité des " corpuscules de Krause ", terminaisons nerveuses que l'on trouve sur les organes sexuels, qui provoqueront « des sensations érotiques nouvelles et presque inouïes » (ibidem, p. 312).

23 Atomized est le titre de la version anglaise des Particules Élémentaires.

${ }^{24}$ M. Houellebecq, Les Particules..., p. 269. 


\section{TOURISTE TERRORISTE}

Plateforme, le troisième roman de Michel Houellebecq, voit son personnage principal Michel, reprendre la proposition de Desplechin au pied de la lettre. Le héros affirme sans ambages dès l'entrée du roman que son seul projet viable est de «pratiquer le tourisme ${ }^{25}$. Lors d'un voyage organisé en Thaillande, il découvre la sexualité pleine d'abandon ${ }^{26}$ de jeunes prostituées locales qui l'enthousiasme. Fort de cette révélation, il imagine remédier à « la névrose occidentale » ${ }^{27}$ par le biais du tourisme sexuel, en échangeant la jeunesse et le capital érotique des habitants des pays pauvres contre la richesse financière des autres. Grâce au mystère ${ }^{28}$ complaisant que représente l'économie globale, l'incel en goguette se trouve par la magie des voyages organisés, propulsé au rang d'homme alpha et peut ainsi avoir accès au vagin $^{29}$ des plus belles femmes pour son seul plaisir. Le programme de Michel est simple, il veut jouir indéfiniment ${ }^{30}$ tout en s'enrichissant, embrassant pleinement les principes du libéralisme moderne. Grâce au tourisme sexuel, les « consommateurs imparfaits $»^{31}$, les homoncules, sont réintégrés dans un système qui jusque-là les excluait. Les rêves éveillés d'orgies internationales tarifées de Michel ne font cependant pas l'unanimité. Le personnage se heurte d'une part à ce qu'il nomme « le courant puritain $»^{32}$, représenté en France par le Guide du Routard : « Des connards humanitaires protestants $»^{33}$ à qui le héros reproche leur hypocrisie sentencieuse ${ }^{34}$. Ces

\footnotetext{
25 Ibidem, p. 34.

26 «[...] si on veut du sexe réel, les pays du tiers-monde» (ibidem, p. 255).

27 Celle-là même qu'il va retrouver à Pattaya (ibidem, p. 361).

28 «L'économie est un mystère » (ibidem, p. 90).
}

${ }^{29}$ Le vagin est la métonymie d'un conflit beaucoup plus large selon Robert, le « beauf » du roman, « Le véritable enjeu de la lutte raciale, articula Robert avec netteté, n'est ni économique ni culturel, il est bio-logique et brutal : c'est la compétition pour le vagin des jeunes femmes » (ibidem, p. 121).

${ }^{30}$ Ce qui correspond chez Bernard Stiegler au « déploiement des tendances pulsionnelles » des sociétés capitalistes qui s'épuisent (B. Stiegler, Mécréance et discrédit, t. $3:$ L'esprit perdu du capitalisme, Galilée, Paris 2006, p. 19).

${ }^{31}$ Nous pensons ici à la définition de Zygmunt Bauman : « Ces gens-là sont les exclus de la société des consommateurs, consommateurs imparfaits, inadéquats et incompétents, en un mot les flops ; des affamés émaciés au milieu de l'opulence du banquet des consommateurs » (Z. Bauman, L'amour liquide, Le Rouergue/Chambon, Cahors 2005, p. 66).

${ }^{32}$ M. Houellebecq, Les Particules..., p. 335.

${ }^{33}$ Ibidem, p. 58.

${ }^{34}$ Cette opposition entre puritanisme anglo-saxon (l'auteur utilise le terme « anglo-saxon » sept fois dans le texte) et hédonisme débridé, est à mettre en rapport avec le dualisme généré par les sociétés modernes : «Les politiques néo-libérales de même que la culture hédoniste-narcissique célébrant le Moi et l'accomplissement immédiat des désirs travaillent parallèlement à dualiser les démocraties, elles génèrent plus de normalisation et plus d'exclusion, plus d'autosurveillance hygiéniste et plus de « défonce » toxicomaniaque, plus de répulsion envers la violence et plus de délinquance dans les ghettos, plus de désir de confort et plus de sans-abri, plus d'amour des enfants et plus de familles sans père » (G. Lipovetsky, L'ère du vide, Gallimard, Paris 2012, p. 321). 
derniers ne représentent qu'une désapprobation symbolique et rapidement évacuée dans le texte. L'autre obstacle à son entreprise, cette fois-ci beaucoup plus conséquent, car générateur de violence possible, est incarné par l'islam et plus précisément par les mouvements islamistes qui sévissent en Asie du Sud-Est. Dans Plateforme, la religion musulmane représente symboliquement et littéralement une limite ; la dernière véritable résistance aux rêves immoraux de libre échangisme de Michel. Cet obstacle potentiel est clairement illustré au début du roman lorsqu'il compare les migrants musulmans qui traversent l'Europe à des caillots « qui se résorbaient lentement $»^{35}$. Les musulmans et de fait l'islam sont de sorte présentés comme un frein potentiel à la société marchande globale, c'est-à-dire à une organisation dont la fluidité toujours plus grande est garante des plaisirs à venir. Pour dénoncer la religion musulmane, l'auteur utilise tout au long du roman une stratégie récurrente qui vise à donner une légitimité à ses attaques : il met en scène des musulman(e)s qui dénigrent l'islam. Au début du roman, le père de Michel est assassiné par un arabe qui est le frère de sa femme de ménage et maîtresse Aïcha. Cette dernière partage avec le héros tout le mépris qu'elle ressent envers les siens. Elle accuse la religion d'avoir des effets délétères sur les hommes de sa famille : «Il y a deux ans, mon père a fait le pèlerinage de La Mecque ; depuis, il n'y a plus rien à en tirer. Mes frères, c'est encore pire : ils s'entretiennent mutuellement dans leur connerie, ils se bourrent la gueule au pastis tout en se prétendant les dépositaires de la vraie foi $[\ldots] »^{36}$. L'auteur utilise ensuite le témoignage d'un Égyptien « biochimiste de formation » ${ }^{37}$, qui se livre à son tour à un réquisitoire enflammé et injurieux contre l'islam. L'Égyptien oppose dans une diatribe enflammée désert et civilisation : le désert représentant la religion musulmane contre la civilisation occidentale. D'après lui, le vide culturel et intellectuel est à l'essence de cette foi dont le lieu de naissance est sa métonymie : « Non, monsieur. L'islam ne pouvait naître que dans un désert stupide, au milieu de bédouins crasseux qui n'avaient rien d'autre à faire — pardonnez-moi - que d'enculer leurs chameaux $»^{38}$. Son argument, bien qu' excessif et insultant, est similaire dans son principe à celui de Desplechin. L'islam comme système de croyances ne peut essentiellement générer aucun progrès intellectuel chez ses disciples, et l'Égyptien de poursuivre : "s'il y a eu des mathématiciens, des poètes, des savants arabes, c'est tout simplement parce qu'ils avaient perdu la foi $»^{39}$. En conclusion du roman, c'est une nouvelle fois un musulman, en l'occurrence un touriste jordanien, qui joint sa voix à la prédiction de Desplechin : «Pour lui il n'y avait aucun doute, le système musulman était condamné : le capitalisme serait le plus fort ${ }^{40}$. Le dualisme qui opposait, dans Les Particules, la religion au progrès scientifique occiden-

\footnotetext{
${ }^{35}$ M. Houellebecq, Les Particules..., p. 30.

36 Ibidem.

${ }^{37}$ Ibidem, p. 260.

${ }^{38}$ Ibidem, p. 261.

${ }^{39}$ Ibidem.

${ }^{40}$ Ibidem, p. 358.
} 
tal glisse ici sémantiquement vers son expression économique la plus conquérante, la société de marché et les valeurs hédonistes qui lui sont associées. Si la sentence reste la même, le pouvoir de nuire de la religion est encore suffisant pour faire dérailler le projet de Michel. À la fin du roman, la compagne du personnage, Valérie, ainsi que de nombreux touristes sont assassinés par un commando d'islamistes. Cet attentat soulève une indignation mondiale qui suscite par association une levée de boucliers contre ce tourisme prédateur. Les Occidentaux dans leur outrage trouvent même des circonstances atténuantes au massacre exécuté par les terroristes : «Le journaliste y accusait carrément le groupe Aurore de promouvoir le tourisme sexuel dans les pays du tiers-monde, et ajoutait que, dans ces conditions, on pouvait comprendre la réaction des musulmans $»^{41}$. Michel Houellebecq, à travers cette justification morale de la violence, met dos à dos deux figures de la modernité : celles du touriste et du terroriste. Le tourisme et le terrorisme sont décrits ici comme des phénomènes internationaux ${ }^{42}$ engendrés par l'économie moderne, l'un justifiant l'autre. L'argument fallacieux du journaliste ${ }^{43}$ est repris indirectement dans la déclaration finale de Michel lui-même. Le tourisme en tant que comportement de masse mortifère et global, reflète une civilisation délétère en pleine dégénérescence, il pourrait expliquer implicitement le terrorisme international :

Jusqu'au bout je resterai un enfant de l'Europe, du souci et de la honte ; je n'ai aucun message d'espérance à délivrer. Pour l'Occident je n'éprouve pas de haine, tout au plus un immense mépris. Je sais seulement que, tous autant que nous sommes, nous puons l'égoïsme, le masochisme et la mort. Nous avons créé un système dans lequel il est devenu simplement impossible de vivre ; et, de plus, nous continuons à l'exporter ${ }^{44}$.

Selon cet aveu qui révèle un dégoût et surtout une culpabilité insoupçonnée jusque là de la part du personnage, le geste des terroristes devient une sorte d'acte salutaire visant à mettre un terme à l'exportation d'un système moralement exécrable qui libère un principe de plaisis ${ }^{45}$ dévastateur. L'islamisme apparaît comme un recours inconscient à la violence déchaînée des désirs funestes occidentaux, ou comme le résume Baudrillard : «À la limite, c'est eux qui l'ont fait, mais c'est

${ }^{41}$ M. Houellebecq, Plateforme, p. 348.

42 « Après quelques minutes de marche dans les rues de Patong Beach, je me rendis compte que tout ce que le monde civilisé avait pu produire en fait de touristes se trouvait réuni là, sur les deux kilomètres du front de mer» (ibidem, p. 111). «Sur la fin ils me montrèrent une série de photos, qui devaient représenter des terroristes internationaux [...]» (ibidem, p. 348).

${ }^{43}$ Un argument qui manque de la connaissance/sincérité la plus élémentaire si l'on considère que la Thaïlande est un pays très majoritairement bouddhiste.

44 Ibidem, p. 348.

45 Selon Michel Clouscard, l'hédonisme est à l'origine du système capitaliste : « Dans le système capitaliste [...] C'est le dressage à la consommation, l'éducation de la "société de consommation" qui sera libérale, permissive et libertaire. C'est la toute-puissance du "principe de plaisir" »" (M. Clouscard, Le capitalisme de la séduction, Delga, Paris 2013, p. 30). 
nous qui l'avons voulu $»^{46}$. La haine de l'islam exprimée à de nombreuses reprises par les personnages du roman s'éclaire d'un jour nouveau. La religion prive ces mêmes protagonistes en souffrance d'une échappatoire possible. Elle donne lieu à une articulation plus subtile du rejet de l'écrivain et annonce ses prises de position à venir. Michel Houellebecq, par le biais de l'islamisme, dénonce la décadence perpétuée dans la société moderne et les violences qu'elle génère. L'islamisme n'est rien d'autre que le pendant religieux de cette frénésie moderne et paradoxalement le moyen opportun dans le récit d'enrayer sa course folle. Car l'écrivain ne verse jamais dans l'essentialisme, les aspirations humaines restent les mêmes chez Houellebecq que l'on soit ou non musulman. L'auteur réalise, par le biais du tourisme sexuel, un distinguo clair entre les musulmans et l'islam ${ }^{47}$. Ces derniers sont, à l'instar des non-musulmans, les victimes doubles de systèmes globaux aux ramifications larges. C'est ainsi que l'auteur dénonce dans la religion musulmane ${ }^{48}$, une violence naturelle, similaire à celle qu'il trouve dans le dogme libéral libertaire ${ }^{49}$. La tentative de son héros de tourner à son avantage et de façon sordide ce phénomène menaçant, se solde par un échec cuisant. La conclusion fataliste et misérable du roman à travers son héros échoué à Pattaya, souligne que l'on ne peut échapper à ces systèmes opprimants, ces cages de fer ${ }^{50}$ indestructibles, avec lesquels il faudra composer ${ }^{51} \mathrm{~d}^{\prime}$ une façon ou d'une autre. Car ces doctrines comptables dangereuses ne visent qu'à faire «payer » aux hommes chacun de leur espoir, aussi minime soit-il. Ou, pour reprendre les mots du touriste égyptien, on ne trouve chez Houellebecq, ni dans la société moderne ni dans l'islam aucune transcendance : «Rien de grand ni de noble, rien de généreux ni de sain ; rien qui puisse faire progresser l'humanité, ni l'élever au-dessus d'elle-même » ${ }^{52}$.

${ }^{46}$ Baudrillard fait référence ici à l'attaque du 11 septembre (J. Baudrillard, « L'esprit du terrorisme », Le Monde, 3 juin 2007, p. 12).

${ }^{47} \mathrm{~S}$ 'ils font preuve du même enthousiasme que les autres à la curée des plaisirs, ils seront considérés eux aussi comme les victimes de ce(s) système(s) impossible(s). De fait, lorsque la population Thaï à la suite d'attentats, manifeste son hostilité à l'égard des touristes musulmans, Michel au contraire ressent pour eux une compassion non feinte : «Les pauvres n'y étaient pourtant pour rien, il était même clair qu'en cas d'attentat ils seraient les premiers visés » (M. Houellebecq, Plateforme, p. 318).

${ }^{48}$ L'auteur, à ce propos, utilise presque uniquement «l'islam » écrit avec une minuscule, c'està-dire à la religion musulmane, et quasiment jamais à « l'Islam » avec une majuscule, la civilisation islamique.

49 Libéral-libertaire est un terme forgé en 1972 par le philosophe et sociologue marxiste Michel Clouscard dans son livre Néo-fascisme et idéologie du désir : Mai 68, la contre-révolution libérale, Delga, Paris 2008. Le terme a donné lieu à de nombreuses controverses.

${ }^{50}$ Cette expression traduite de l'allemand stahlhartes Gehäuse, souligne à l'origine chez Max Weber, l'oppression d'un système bureaucratique basé sur le calcul et le contrôle des individus. Elle s'applique chez Houellebecq dans son aspect coercitif global (M. Weber, L'Éthique protestante et l'esprit du capitalisme, Gallimard, Paris 2003, p. 251).

${ }^{51}$ Nous pensons ici à l'injonction de Charles Taylor « Nous ne sommes pas enfermés. Mais il existe une pente, une inclinaison dans les choses à laquelle il est trop facile de se laisser aller » (C. Taylor, Le malaise de la modernité, Les Éditions du Cerfs, Paris 2005, p. 107).

52 M. Houellebecq, Les Particules..., p. 263. 


\section{TRANSITION IMPOSSIBLE}

La Possibilité d'une île est un roman de transition du point de vue de la religion musulmane. Pour cette raison il sera traité brièvement dans cette étude. Il permet à Michel Houellebecq de mettre en scène la "prophétie » faite dans ses ouvrages précédents. Dans un premier temps, l'islam fait illusion et prend la place laissée vacante par la religion chrétienne en France et dans les pays occidentaux. Elle offre aux Européens cette cohésion sociale disparue, qui inquiétait tant les protagonistes des Particules:

En l'espace d'une à deux décennies, l'islam devait ainsi parvenir à assumer en Europe le rôle qui était celui du catholicisme au cours de sa période faste : celui d'une religion « officielle », organisatrice du calendrier et des mini-cérémonies rythmant le passage du temps, aux dogmes suffisamment primitifs pour être à la portée du plus grand nombre $[\ldots]^{53}$.

Mais le charme ne va pas durer. Selon la lecture causaliste de l'écrivain, l'islam suivra le même chemin que le catholicisme et le communisme avant lui. Le théorème eschatologique houellebecquien est simple : "Lorsqu'un système social est détruit, cette destruction est définitive, et aucun retour en arrière n'est possible $[\ldots]{ }^{54}$. Cette chute brutale annoncée, confirme le point de vue de Desplechin comme ceux des protagonistes musulmans de Plateforme : l'islam est bien une religion rétrograde et violente : « [...] les pays musulmans n'avaient été maintenus dans leur foi primitive que par l'ignorance et la contrainte ; privés de leur base arrière, les mouvements islamistes occidentaux s'effondrèrent d'un seul coup $\rangle^{55}$. Mais la conclusion du roman infirme aussi son postulat matérialiste et scientifique. Daniel 25, le dernier clone du roman, réalise qu'il n'existe pas d'issue magique à la souffrance humaine : «J'étais indélivré » ${ }^{56}$. Michel Houellebecq met un terme dans La Possibilité à ses spéculations post-humaines. Dépourvu de transcendance, l'homoncule devra trouver une échappatoire immanente ${ }^{57}$ à sa situation à travers la vision opportuniste d'un islam finalement taillée sur mesure.

\section{ISLAM IMMODÉRÉ}

Dans son roman Soumission, Michel Houellebecq propose une interprétation de l'islam qui correspond parfaitement aux attentes de son personnage François. Son héros est au début du roman, le témoin privilégié de remous politiques et sociaux qui accompagnent une élection présidentielle troublée. Elle oppose dans le futur proche, le Front national de Marine Le Pen à la Fraternité musulmane de

\footnotetext{
${ }^{53}$ M. Houellebecq, La Possibilité d'une île, p. 358.

54 Ibidem.

${ }^{55}$ Ibidem, pp. 359-360.

56 Ibidem, p. 484.

${ }^{57} \mathrm{Si}$ l'on excepte la littérature, mais ce n'est pas le propos de cette étude.
} 
Mohamed Ben Abbes. François décide de laisser Paris pour trouver dans la France du Sud-Ouest un refuge momentané. C'est lors de cet antivoyage initiatique qu'il prend conscience de l'importance de la civilisation chrétienne médiévale dans l'histoire de la civilisation française. Il mesure aussi tout le gouffre qui le sépare désormais de cette tradition. Un gouffre selon lui creusé plus profondément encore par la lecture postrévolutionnaire et romantique qu'en fit le $\mathrm{XIX}^{\mathrm{e}}$ siècle. La perspective moderne s'en trouve faussée comme l'illustre la critique qu'il fait de son mentor Joris-Karl Huysmans ${ }^{58}$. Cette réalisation faite par François à la suite de sa visite de la grotte de Rocamadour, l'éloigne de tout retour possible vers un christianisme dorénavant honni. Car selon le héros, non seulement il ne reste rien de la foi chrétienne des hommes médiévaux, mais en plus, la religion catholique s'est compromise de façon avilissante avec la société mercantile moderne. Ainsi, la mort de la religion chrétienne en Europe annonce, comme l'auteur l'avait précédemment développé dans Les Particules, la fin inéluctable de la société française et de l'occident tout entier :

À force de minauderies, de chatteries et de pelotage honteux des progressistes, l'Église catholique était devenue incapable de s'opposer à la décadence des mœurs. De rejeter nettement, vigoureusement, le mariage homosexuel, le droit à l'avortement et le travail des femmes. Il fallait se rendre à l'évidence : parvenue à un degré de décomposition répugnant, l'Europe occidentale n'était plus en état de se sauver elle-même ${ }^{59}$.

En écho à son roman Les Particules Élémentaires, qui focalisait sa critique de la religion sur la montée d'un éthos scientifique désormais indépassable dans le monde moderne, c'est plus précisément à l'incorporation des règles du libertarisme à la religion elle-même, que s'attaque Soumission. La religion musulmane dans un tel contexte apparait, en écho au terrorisme de Plateforme, comme une alternative salvatrice. C'est ainsi que Michel Houellebecq prépare son lecteur aux changements que l'islam politique va entraîner dans la société française à la suite de l'élection surprise de son représentant, Mohamed Ben Abbes. Pour comprendre toute la portée de ces changements dans l'existence de son personnage, il nous faut apprécier la nouvelle version du mâle dominé qu'est François dans le roman. Le personnage n'est plus exactement la victime expiatoire et malheureuse rencontrée dans les romans précédents. François se distingue des autres homoncules, en se présentant comme un consommateur exigeant et aguerri qui engloutit force de nourritures, achète quantité de produits, et choisit sur internet avec expertise les prostituées qui lui conviennent. Il n'est plus tout à fait non plus l'incel esseulé et frustré des premiers textes. Si le héros vieillissant doit faire face à la solitude, c'est qu'il se l'est imposée par un mélange paradoxal de fatalisme et d'apathie. Il a en effet la possibilité de vivre une véritable histoire d'amour

${ }^{58}$ Le personnage prend conscience de cette réalité en découvrant la vierge noire de Rocamadour : « Il y avait là quelque chose de mystérieux, de sacerdotal et de royal que Péguy n'était pas en état de comprendre, et Huysmans encore bien moins » (M. Houellebecq, Soumission, p. 170).

${ }^{59}$ Ibidem, p. 275. 
avec son ex-étudiante Myriam, mais il demeure sans réaction lors de son départ : « je pris alors douloureusement conscience que je n'avais même pas proposé à Myriam de venir habiter chez moi, de s'installer ensemble ${ }^{60}$. Il justifie son comportement en arguant de la perte inévitable du désir qui rend tout projet de vie à deux obsolète. L'origine de cette pensée débilitante qui paralyse l'homoncule houellebecquien ${ }^{61}$ est à trouver dans le modèle économique qui s'impose à tous les niveaux de la société. Ce nouveau paradigme éreinte ses participants : "Les héros de la consommation sont fatigués " ${ }^{62}$, il sous-tend un pessimisme et un cynisme de tous les instants qui participe à cet abattement général. Il s'explique précisément dans le principe entropique des sociétés capitalistes, thème récurrent dans l'œuvre de l'écrivain ; sociétés qui tôt ou tard voient leur profit s'effondrer : « Au bout de la concurrence, le profit est nul — grand principe économique. À la baisse tendancielle du taux de profit, ajoute Michel Houellebecq, correspond la baisse tendancielle du taux de désir : cette société ne sait plus comment attiser le désir, exciter les sens $»^{63}$. Ce modèle économique conditionne le comportement amoureux de François borné à une perspective de consommation pure. Il reconnaît ainsi que toute vie de couple est condamnée à l'avance, car elle est tributaire non plus de normes sociales à la fois contraignantes et protectrices comme dans la société bourgeoise traditionnelle, mais du désir fluctuant qui est par définition impossible à satisfaire dans l'économie de marché, suivant son principe le plus essentiel :

La société de consommation promettait de satisfaire les désirs humains d'une façon qu'aucune autre société ne fut jamais capable de concevoir (pas même en imagination). Cette promesse de satisfaction ne demeure toutefois séduisante que tant que le désir reste insatisfait ; et surtout, tant que subsiste le soupçon que le désir n'a pas été vraiment, entièrement, satisfait ${ }^{64}$.

Pour résoudre cette aporie dévastatrice, Michel Houellebecq invente dans Soumission une version de l'islam qui permet d'éviter cet écueil. L'auteur va combiner à cet effet certaines prérogatives revenant à l'homme musulman en les associant aux privilèges du consommateur jouisseur moderne. Il recycle ainsi ces deux doctrines, en les mettant « au service» de ses personnages masculins. Il focalise de fait son propos sur une application mercantile et politique ${ }^{65}$ de la religion. L'auteur invente dans son roman un matérialisme islamique bienveillant, incarné par le personnage

60 Ibidem, p. 113.

${ }^{61}$ Cette paralysie affecte de nombreux héros, Jed dans La Carte et le Territoire, se déclare pareillement « trop lâche ou trop indécis » (M. Houellebecq, La Carte et le Territoire, Flammarion, Mayenne 2010, p. 251)

62 J. Baudrillard, La société de consommation, Denoël, Paris 1970, p. 292.

63 B. Maris, Houellebecq économiste, Flammarion, Mayenne 2015, p. 125.

${ }^{64}$ Z. Bauman, La vie liquide, Éd. Christophe Rosson, Rodez 2006, p. 105.

65 « La vraie question désormais [...] est celle des ressources que comportent l'islam pour s'adapter à une modernité politique qui s'est construite en dehors de lui » (M. Gauchet, La Condition Historique, Éditions Stock, Paris 2003, p. 164). 
paradoxal de Mohamed Ben Abbes ${ }^{66}$. L'homme politique musulman est une personnalité mystérieuse qui va rendre possible cette prémisse surprenante. Grâce à lui, le héros va pouvoir envisager une nouvelle stratégie de consommation et espérer, en conclusion de l'ouvrage, satisfaire tous ses désirs ${ }^{67}$. Ben Abbes est présenté comme un leader exceptionnel et paradoxal, il est paré de toutes sortes de qualités et de vertus : il est « enjoué $~^{68}$ et $~ «$ malicieux ${ }^{69}$ et $«$ extrêmement habile $»^{70}$. C'est surtout un pur produit de 《 la méritocratie républicaine ${ }^{71}$, il fut « un des plus jeunes polytechniciens de France avant d'intégrer l'ENA $»^{72}$. Cette dernière information souligne la singularité d'un personnage musulman d'origine arabe et qui incarne par ailleurs l'excellence de la culture française. C'est ainsi qu'il prétend apporter à la société : « un nouvel humanisme [...] réunificateur $»^{73}$ qui s'inspire du Coran et qui s'oppose fondamentalement aux valeurs post-religieuses occidentales comme « le sécularisme, la laïcité, le matérialisme athée ${ }^{74}$. Ben Abbes fait le lien dans sa politique réactionnaire entre l'Ancien régime et la société contemporaine en se débarrassant de l'héritage idéologique progressiste de la révolution française. Un tel programme reçoit l'adhésion des Français dans le roman, car nous apprend François : «C'est un musulman modéré [...] $»^{75}$. Cette étiquette rassurante lui permet d'être accepté par les partis politiques de tous bords, si l'on omet l'extrême droite. Du point de vue de la narration, il est important que Ben Abbes soit un homme exceptionnel, cette singularité s'inscrit dans la logique de soumission du peuple français du futur qui accepte par le biais de cet homme hors du commun, de suivre les préceptes d'un régime théocratique sans se révolter ${ }^{76}$. La politique économique intérieure de Ben Abbes est elle aussi surprenante, c'est un compromis de religion et de justice sociale. Elle se situe sur une ligne traditionaliste mâtinée de socialisme tribal. Le politicien déclare s'inspirer du distributivisme, une troisième voie entre capitalisme et communisme :

Son idée de base était la suppression de la séparation entre le capital et le travail. La forme normale de l'économie y était l'entreprise familiale ; lorsqu'il devenait nécessaire, pour certaines produc-

${ }^{66}$ Ben Abbes est avant toute chose un marchand, comme l'illustre son héritage : « Il évoquait plutôt un bon vieil épicier tunisien de quartier — ce que son père avait été d'ailleurs » (M. Houellebecq, Soumission, p. 108).

${ }^{67}$ La conversion n'est qu'une hypothèse dans le roman. L'auteur utilise pour la décrire le mode conditionnel et jamais le présent ni le passé de l'indicatif.

${ }^{68}$ M. Houellebecq, Soumission, p. 108.

${ }^{69}$ Ibidem.

70 Ibidem, p. 154.

71 Ibidem, p. 108.

72 Ibidem.

73 Ibidem, p. 152.

74 Ibidem, p. 156.

75 Ibidem, p. 154.

${ }^{76}$ Il est évident que la « soumission » du peuple français est aussi un des arguments du roman pour dénoncer une société en déliquescence. 
tions, de se réunir dans des entités plus vastes, tout devait être fait pour que les travailleurs soient actionnaires de leur entreprise, et coresponsables de sa gestion ${ }^{77}$.

Le distributivisme n'est pas propre à la religion musulmane, c'est au contraire une philosophie économique d'inspiration catholique ${ }^{78}$. C'est une façon habile de la part du politicien, d'inscrire cet islam politique dans la droite ligne d'une pensée sociale chrétienne, familière à la tradition française. Mais ce sont surtout ses applications domestiques qui vont intéresser le héros. Car cette philosophie réactionnaire insiste essentiellement sur le renforcement de la cellule familiale : « le cadre chaleureux de la cellule familiale $»^{79}$. La famille, selon le narrateur, est devenue un projet quasiment impossible dans le système libéral moderne comme nous l'avons souligné. La compétition dans le monde du travail épuise les couples et brise les passions ${ }^{80}$, car elle oppose brutalement les sexes. Comme une sorte d'antidote à cette situation déprimante, François fantasme sur un exotisme érotique propre à l'islam. Il rêve à des femmes musulmanes, ici de riches Saoudiennes, qu'il imagine lascives et entièrement dévouées à leurs hommes : « (elles) se transformaient le soir en oiseaux de paradis, se paraient de guêpières, de soutiens-gorge ajourés, de strings ornés de dentelles multicolores et de pierreries ; exactement l'inverse des Occidentales ${ }^{81}$. Cet argument caricatural nous renseigne sur le type d'islam conçu dans le roman ; c'est une version contemporaine de l'orientalisme qui se rapproche plus des tableaux de Jean-Léon Gérôme ${ }^{82}$ que d'une quelconque réalité adaptable à la société française. Or, les chimères du personnage vont trouver dans les réformes politiques de Ben Abbes un écho inespéré. Ce dernier veut renforcer les familles en détournant les femmes du monde du travail, pour les ramener au foyer : «Ce qu'ils souhaiteraient au fond c'est que la plupart des femmes, après l'école primaire, soient orientées vers des écoles d'éducation ménagère, et qu'elles se marient aussi vite que possible [...] ce serait leur modèle de société idéal ${ }^{83}$. Le héros est involontairement comblé par ce projet, car s'il perd dans un premier temps un peu du plaisir voyeuriste qu'il avait à observer le corps des femmes déambulant dans la rue ${ }^{84}$, il peut prétendre, s'il se convertit à l'islam, combler son désir mimétique ${ }^{85}$, c'est-à-dire posséder plusieurs épouses au service exclusif de son plaisir. Cet idéal phallocrate est originellement exprimé

${ }^{77}$ M. Houellebecq, Soumission, p. 202.

${ }^{78}$ Cette philosophie économique fut développée par des penseurs catholiques tels Gilbert Keith Chesterton ou Hilaire Belloc.

${ }^{79}$ M. Houellebecq, Soumission, p. 210.

${ }^{80}$ Voir l'épisode de la soirée ratée de Bruno et Annelise Deslandes, pp. 90-94.

${ }^{81}$ M. Houellebecq, Soumission, p. 91.

${ }^{82}$ L'auteur mentionne un tableau de Bouguereau dans le roman qui comme Gérôme fut un des maîtres de l'académisme.

${ }^{83}$ M. Houellebecq, Soumission, p. 82.

84 « La contemplation du cul des femmes, minime consolation rêveuse, était elle aussi devenue impossible » (ibidem, p. 177).

85 J'utilise ce terme ici en rapport avec la relation qu'il entretient avec Huysmans. 
par Huysmans dans le roman à travers la caractérisation de deux femmes-objets : la « femme pot-au-feu » et la « fille ${ }^{86}$. Ces deux caractères apparaissent en personne à François vers la fin du roman, lorsqu'il rencontre les épouses musulmanes du converti Rediger. Il y a la très jeune Aïcha qui représente du fait de son jeune âge, littéralement la fille. C'est une sorte de Lolita musulmane « une fille d'une quinzaine d'années, vêtue d'un jean taille basse et d'un tee-shirt Hello Kitty ${ }^{87}$ " . Il y a ensuite Malika la femme-pot-au-feu : " une femme d'une quarantaine d'années, grassouillette et d'allure bienveillante, apparut, portant un plateau sur lequel étaient disposés des petits pâtés chauds et un seau à glace contenant la bouteille de Meursault promise $»^{88}$. Cette version caricaturale et opportuniste de la religion musulmane permettrait à l'homoncule, grâce au mariage arrangé, d'échanger une vie plus ou moins solitaire et promise à des frustrations toujours plus grandes, pour une existence familiale confortable et pleine de plaisir. L'avantage de cette version de l'islam instaurée par Ben Abbes, c'est que ces nouvelles prérogatives s'appliquent à tous les incels du roman sans exception. Ils appartiennent exclusivement à la catégorie des clercs, c'est-à-dire des intellectuels de l'université pour laquelle travaille François. Son ancien collègue Steve, universitaire médiocre et frustré, annonce avec un grand sourire à François : « Je vais prendre une deuxième épouse le mois prochain ${ }^{89}$. Plus surprenant encore est le personnage de Loiseleur, professeur de littérature et spécialiste du XIX ${ }^{\mathrm{e}}$ siècle comme François. Il est décrit dans l'ouvrage comme l'archétype de l'homme bêta : " cheveux longs, gris et sales, lunettes de vue énormes, costumes dépareillés et dans un tel état qu'il paraissait souvent à la limite de l'hygiène $»^{90}$. Loiseleur est le premier surpris à la suite de sa conversion à l'islam de pouvoir vivre avec une femme : "Oui oui, une femme, ils m'ont trouvé ça ${ }^{91}$. Voilà le tour de force de Michel Houellebecq dans Soumission, détourner le spectre de l'islam au profit d'un libéralisme réactionnaire dépourvu de tout libertarisme. À force de tâtonnements et de scénarios plus ou moins fantastiques, il semblerait que l'homoncule soit enfin sauvé, grâce à la religion musulmane...

Michel Houellebecq invente dans ses romans une foule de subterfuges plus invraisemblables les uns que les autres, pour tenter de protéger les perdants d'un système et atténuer leurs souffrances respectives. Dans un tel scénario, l'islam est perçu à l'origine, comme une menace lointaine vis-à-vis des rêves hédonistes de ses losers. Le danger se précise ensuite pour se transformer en rappel à l'ordre violent, quand la complaisance érotique et vénale de son personnage génère en Thaïlande, une application cynique et opportuniste à son désir. Le péril de l'islam

\footnotetext{
${ }^{86}$ M. Houellebecq, Soumission, p. 97.

${ }^{87}$ Ibidem, p. 243.

${ }^{88}$ Ibidem, p. 247.

${ }^{89}$ Ibidem, pp. 181-182.

${ }^{90}$ Ibidem, p. 286.

${ }^{91}$ Ibidem, p. 288.
} 
est finalement détourné et récupéré dans Soumission, au profit des mâles dominés qui s'en trouvent transformés. Dans ce nouveau système, Rediger le bonimenteur apprend à François l'homoncule qu' « il n'y a rien d'anormal que les professeurs d'université soient rangés parmi les mâles dominants ${ }^{92}$. La religion musulmane chez Michel Houellebecq s'accorde miraculeusement avec l'hédonisme individualiste prôné par la société libérale. L'écrivain invente à cette fin, une idéologie hybride taillée sur mesure pour son héros esseulé ; c'est un idéal de consommation ininterrompue mâtiné de traditionalisme religieux machiste. Mais c'est un libéralisme toutefois affaibli qu'invente Houellebecq, car il est largement épuré de son principe de compétition. Cette idéologie paradoxale encourage et stimule de fait les " possibilités vivantes ${ }^{93}$ des anciens exclus du banquet des consommateurs ${ }^{94}$. Cependant, et c'est l'argument de notre recherche, l'écrivain n'est jamais dupe des fables qu'il imagine ; chaque postulat mis en place dans ses romans s'effondre immanquablement, et l'islam n'échappe pas à cette règle. La religion est d'abord amoindrie dans sa dimension spirituelle, elle est dépourvue de la plus simple transcendance à l'image des deux figures qui la représente dans le texte : avec d'une part son leader politique charismatique, le fils d'épicier Mohamed Ben Abbes et d'autre part l'opportuniste converti, l'impétrant manipulateur Robert Rediger. La religion ne représente plus, à la fin de l'ouvrage, qu'une hypothèse aussi caricaturale qu'improbable, une nouvelle proposition impossible à mettre au conditionnel. Le mode hypothétique ${ }^{95}$ de la conclusion est un rappel subtil de l'écrivain à son lecteur : il nous enseigne que le monde, malgré nos désirs, n'est ni un panorama ${ }^{96}$ ni un supermarché géant qui viserait in fine à détourner notre conscience ${ }^{97}$. Il est au contraire tragique, car l'homme moderne est prisonnier d'un matérialisme sans issues, et à la fin tout sera à regretter ${ }^{98}$. Dans ces conditions, l'islam et ses applications politiques ne sont que le moyen utilisé par l'auteur pour affirmer le caractère supérieur et transcendant ${ }^{99}$ de « l'art majeur $\aleph^{100}$ qu'est la littérature. Une excellence artistique qui prime sur toutes les autres formes d'expression et de pensée. En écho à son apologie de la littérature

92 Ibidem, p. 292.

93 Ibidem, p. 204.

94 Voir la note 30.

95 Voir la note 66.

96 « Le monde n'est pas un panorama », note sèchement Schopenhauer » (M. Houellebecq, La Possibilité d'une île, p. 109).

97 «Et la consommation perpétuelle n'est-elle pas la forme suprême du divertissement pascalien ? Oublier que le temps passe et que la mort approche, en mangeant, en changeant d'objet ?» (B. Maris, op. cit., p. 74).

${ }^{98}$ Le roman se conclut par cette antiphrase : « Je n'aurais rien à regretter » (M. Houellebecq, Soumission, p. 300).

99 «Seule la littérature peut vous permettre d'entrer en contact avec l'esprit d'un mort » (ibidem, p. 13).

100 Ibidem. 
qui inaugure son roman, l'écrivain met en scène cette ultime croyance avec une grande ironie en conclusion de son texte. Il choisit le centre historique des lettres françaises, la Sorbonne, pour décrire un spectacle absurde et grand-guignolesque supposé peindre la conversion fantasmatique de François entouré de ses collègues, les clercs humiliés, affublés de toges ${ }^{101}$. L'auteur réalise ainsi à un ultime pied de nez littéraire qui parachève la suprématie du « vers » sur celle du verset ${ }^{102}$, ou du roman sur le texte sacré.

\section{ISLAM IN MICHEL HOUELLEBECQ’S NOVELS}

\section{Summary}

Michel Houellebecq's take on Islam changed drastically through the years. He first described it as the remnant of an antiquated order, based on superstition and backward principles. He then used Islamic terrorism as a violent limit to its character's complacent plight for pleasure. But his denunciation of Islam extremism is accompanied by a stronger condemnation of capitalism. Submission is Houellebecq's ultimate opportunity to combine and transform two dreaded ideologies into one benevolent system that could potentially benefit his hero, the ill-fated homunculus.

Key words: homonculus, Islam, materialism, transcendence, literature.

101 « [...] les autorités saoudiennes avaient récemment rétabli le port de ce vêtement d'apparat » (ibidem, p. 299).

102 Concept développé par Kamel Daoud dans son roman Zabor, Actes Sud, Alger 2017. Et repris dans l'émission de France Culture, «Le temps des écrivains », <https://www.franceculture.fr/ emissions/le-temps-des-ecrivains/emission-speciale-kamel-daoud $>$ [consulté le 30.08.2018]. 